\title{
MICROINSTRUMENT FOR TRANSDERMAL, WOUNDPOTENTIAL MEASUREMENTS
}

\author{
E. V. Mukerjee, S. D. Collins, and R. L. Smith \\ Micro Instrument and Systems Laboratory (MISL) \\ Department of Electrical \& Computer Engineering \\ University of California, Davis \\ Davis, California, 95616
}

\author{
R. R. Isseroff \\ Department of Dermatology \\ University of California, Davis \\ Davis, California, 95616
}

\author{
R. Nuccitelli \\ Department of Molecular and Cellular Biology \\ University of California, Davis \\ Davis, California, 95616
}

\section{ABSTRACT}

In vivo animal studies have shown that electrical fields radiate laterally from skin wound sites [1]. In addition, in vitro testing of keratinocytes (the first cellular response to wound healing) has shown cathodally-directed migration in physiological DC electrical fields (100-200 $\mathrm{mV} / \mathrm{mm})$ [2]. Results from human wound studies are less forthcoming due to mechanical fragility inherent with standard drawn glass electrode probes. An MEMS alternative was therefore pursued as a reliable, repeatable, wound potential measuring device.

This paper presents the design, fabrication and preliminary testing of an individually addressable, micro-needle array for the measurement of endemic electric fields in wounded tissue. Recording sites are defined by photolithographically patterning thin film Pt electrodes onto a glass substrate, which end in an array of electrical contacts [5]. Aligned anodic bonding of silicon to glass and subsequent dicing with a diamond embedded saw blade create silicon columns attached as an array to both the glass substrate, and the thin film electrodes. The outcome is an array of silicon micro-needles bonded to an electrically insulating glass substrate with $\mathrm{Pt}$ electrodes addressing individual needles. Wet chemical etching (HNA) [3,4] sharpens the columns into needles that are sharp enough to pierce skin yet robust enough to withstand multiple penetrations with minimal tip damage (Figure 1). Using a $38.7 \mathrm{mV}$ sinusoidal input, an input/output separation distance of 6 inches and a $1 \mathrm{M} \Omega$ resistor in parallel, an output signal of 18 $20 \mathrm{mV}$ was measured by the micro-needle array.

\section{METHODS}

\subsection{Silicon substrate}

The needles were fabricated in 4 inch diameter, (100) oriented, p+, silicon wafers. Aluminum was used as a dry etch mask and was deposited onto the polished silicon surface using a CHA Industries electron beam evaporator. Standard photolithography and aluminum etching opened pattems for silicon etching by Reactive Ion Etching (RIE). The patterned aluminum wafer was subsequently etched in a Technics MicroRIE using sulfur hexafluoride ( $\mathrm{SF}_{6}$ ) and oxygen (15 and $1.4 \mathrm{sccm}$, respectively) at 400 watts to a final depth of $18.5 \mu \mathrm{m}$. An orthogonal set of trenches, $150 \mu \mathrm{m}$ deep, on $300 \mu \mathrm{m}$ centers, was made into the etched surface with a wafer dicing saw (MicroAutomation I006), creating an array of silicon pillars. The aluminum was then removed using a wet etchant (PAN) followed by a BOE dip to remove any silicon dioxide from the silicon chip surface.

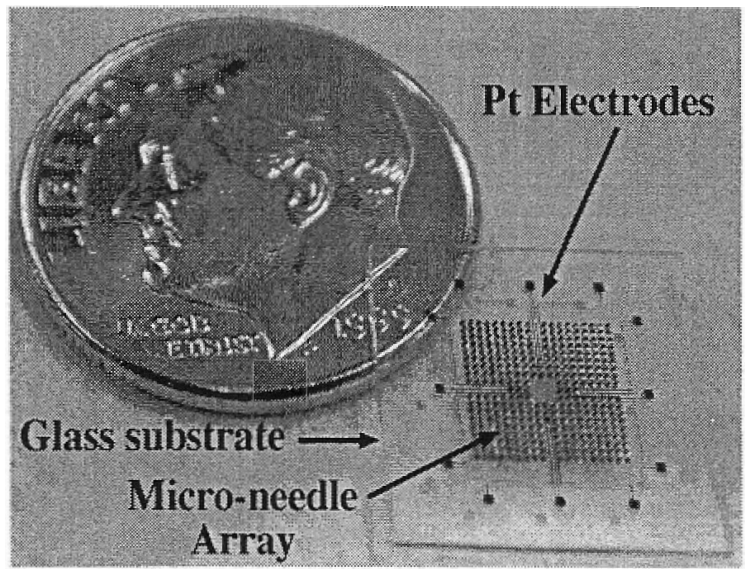

Figure 1. Wound Potential Device

\subsection{Glass substrate}

Pyrex 7740 glass wafer was used as a platform for the electrodes. Normal photolithographic procedures were used to pattern photoresist on the glass surface as a lift-off mask for the thin film electrode array. The wafer was dipped in a 50:1 HF solution prior to being placed in the CHA Electron beam evaporator (E-beam) for tantalum (Ta) and platinum $(\mathrm{Pt})$ deposition ( $100 \AA$ and $500 \AA$, respectively). The $\mathrm{Pt}$ was then patterned by lift-off of the photoresist in organic solvent.

\subsection{Anodic Bonding}

The silicon and glass wafers were aligned so as to position the Pt electrodes between the glass and silicon and the contact pads directly beneath the appropriate silicon pillars. Once manually aligned, the bonding cathode was brought down onto the glass surface, adding enough pressure to ensure contact throughout the bonding process. Bonding was performed at $425^{\circ} \mathrm{C}$, and $1000 \mathrm{~V}$ for 1 minute.

\subsection{Needle fabrication}

The silicon top surface of the bonded pair was coated with photoresist and hard baked for $>1$ hour. With the glass side down on the dicing saw, cuts were made in the silicon surface, to a depth of about $350 \mu \mathrm{m}$. This resulted in an array of silicon $3 \mathrm{D}$ structures 
with a $200 \mu \mathrm{m}$ square base, and $150 \mu \mathrm{m}$ tall, on top of which sits a $350 \mu \mathrm{m}$ tall, silicon, rectangular column with a $100 \mu \mathrm{m}$ square cross-section. The silicon structures were then etched in "HNA" (1:8:1 hydrofluoric: acetic: nitric acids) until the desired needle shape was obtained. The over baked photoresist on the tops of the columns protected the surface long enough to sharpen them (at which point they fall off) and helped produce needles of uniform height. The chip was then rinsed and oven dried. The needles were then punctured through aluminum foil $(-100 \mu$ m thick $)$ until the tips of the needles were exposed. The assembly was place in the E-beam (tips towards the source) for deposition of $100 \AA \mathrm{Ta}$ and $500 \AA \mathrm{Pt}$. The foil acted as a shadow mask, protecting all underlying structures from metal deposition. The aluminum foil was removed leaving metal only at the tips of the needles. The entire chip was then coated with $1000 \AA$ of PECVD silicon nitride (Technics) and subsequently etched by an RIE using $\mathrm{CF}_{4} / \mathrm{O}_{2}$ (15 sccm) and $200 \mathrm{~W}$ until the silicon nitride was removed from the tips of the needles [6] (Figure 2).

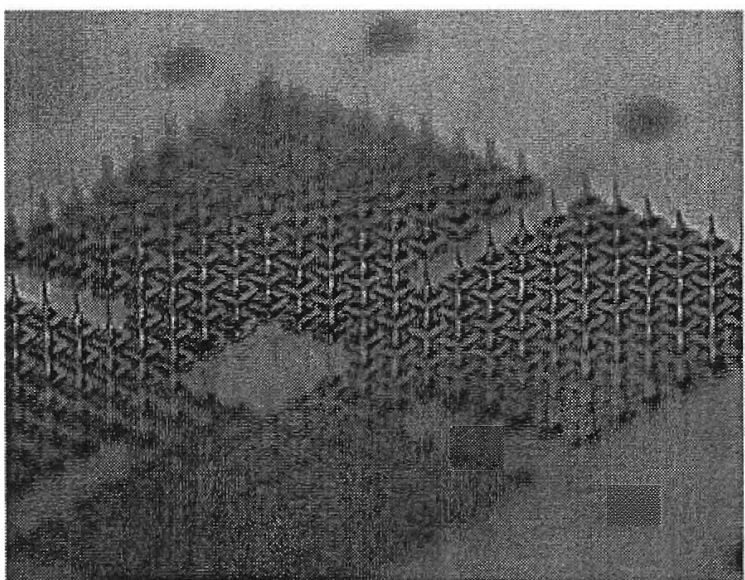

Figure 2. Completed array showing etched silicon microneedles and electrodes attached to a glass substrate.

\section{EXPERIMENTATION AND RESULTS}

\subsection{Resistance measurements}

After the initial bonding and before the second set of dicing cuts were made, contact resistance was measured. A Fluke 8060A Digital Multimeter was used. Three resistance measurements were made: 1) the surface of the silicon $-45 \Omega \quad 2$ ) the electrical contact pad and the silicon --280 $\Omega$ 3) electrical contact pad to another electrical contact pad (using the silicon as the connection between them) $--735 \Omega$.

\subsection{In vitro resistance measurements}

A saline solution at physiological concentrations was used to test individual addressing and isolation of contacted needles. A stainless steel reference electrode and the tips of the micro-needle array, connected by a Fluke 8060 A Digital Multimeter, were place into the solution and resistance measurements were taken between individual electrodes when their tips were in versus out of the saline solution. Out of solution, the needles were an open circuit (isolation). In the saline, a resistance of $430 \mathrm{k} \Omega$ was measured.

\subsection{In vivo ECG measurements}

Using a Medi-Trace 1 S'Offset ECG $\mathrm{AgCl}$ as a source electrode, a $38.7 \mathrm{mV}$ sinusoidal signal was introduced into the system. Measurements of the signal were taken by the microneedle array placed 6 inches from the ECG electrode along the subject's forearm. A $1 \mathrm{M} \Omega$ resistor was placed from the microneedles to ground decrease the current through the subject's tissue. $18-20 \mathrm{mV}$ was measured by a single micro-needle. Using the same arrangement, a sub-dermal DC resistance value of $613 \mathrm{k} \Omega$ was calculated.

\section{SUMMARY}

Utilizing MEMS technology, a transdermal micro-needle array, with individually addressable needles, was fabricated and bench tested. It was shown that the device has the mechanical strength to puncture the skin and the electrical conductivity to measure both resistance and potential differences both in vitro and in vivo. These measurements lay the groundwork for further testing, specifically the recording of biopotentials related to wound generation and healing.

\section{AGKNOWLEGMENTS}

The authors would like to express our gratitude to Dwight Howard for his expertise and on-going technical support throughout the duration of this project.

\section{REFERENCES}

[1] D. Inglesia, E. Cragoe, Jr., and J. Vanable, Jr., "Electric field strength and epithelization in the newt (Notophthalus viridescens)", Journal of Experimental Zoology, 274 (1996)

[2] K. Y. Nishimura, R. R. Isseroff, and R. Nuccitelli, "Human keratinocytes migrate to the negative pole in direct current electric fields comparable to those measured in mammalian wounds", Journal of Cell Science 109 (1996)

[3] S. K. Ghandi, VLSI Fabrication Principles-silicon and gallium arsenide, John Wiley and Sons, New York 1994

[4] P. Campbell, K. Jones, R. Huber, K. Horch, and R Normann, "A silicon-based, three-dimensional neural interface: manufacturing processes for an intracortical electrode array", IEEE Transaction on Biomedical Engineering, 38, 8 (1991)

[5] S. Tatic-Lucic, J. Ames, B. Boardman, D. McIntyre, P. Jaramillo, L. Starr, and M. Lim, "Bond-quality characterization of silicon-glass anodic bonding", Sensors and Actuators A 60 (1997)

[6] G. Schurmann, P.F. Indermuhle, U. Stauer, and N.F. de Rooij, "Micromachined SPM probes with sub-100 nm feature at tip apex", Proceedings of the 3rd Conference on Development and Industrial Application of Scanning Probe Methods (SXM-3), Basel, Switzerland, 9/I6-19 (1998) pp.299-301 